Chapter 8

\title{
Laser Ablation in Different Environments and Generation of Nanoparticles
}

\author{
Abubaker Hassan Hamad, \\ Khawla Salah Khashan and Aseel Abdulkreem Hadi
}

Additional information is available at the end of the chapter

http://dx.doi.org/10.5772/65241

\begin{abstract}
In the last two decades, pulsed-laser ablation has received attention from researchers in micro- and nanotechnology. During the development of laser ablation in materials processing, several media, such as vacuum, air, gases and liquids, have been used to improve the quality and quantity of laser machining and production of nanoparticles. The laser-ablation environment is important in order to control the average size and chemical compositions of nanoparticles. Conducting the laser-ablation process in liquid environments has become of increasing relevance for the production of precise and pure micromachining and nanomaterials. In addition, deionised water has been found to be the optimal environment to produce nanoparticles for bioapplications.
\end{abstract}

Keywords: laser ablation, vacuum, air, liquids, gases, nanoparticles, ablation mechanism, productivity

\section{Introduction}

Different pulsed-laser ablations of the solid target materials have shown great potential in the fields of laser-material microprocessing, nanotechnology and device fabrication. To develop the quality and quantity of micro- and nanomachining, laser ablation of materials has been carried out in different environments, such as in a vacuum [1], in air [2], in gases [3] and in liquids [4] for different applications such as welding [5], cladding [6], cutting [7], cleaning [8] and generation of nanoparticles. 
Although some laser beam parameters such as laser fluence, wavelength and pulse duration are important for controlling material processing, the ablation environment is also an important factor in laser-material interaction. For example, Zhu et al. [9] concluded that the ablation rate of a Si target material is greatly enhanced by using a water confinement regime (WCR) at laser fluence ranges from 2.0 to $5.0 \mathrm{~J} / \mathrm{cm}^{2}$. They also found that in water, the first peak-to-peak amplitude of the acoustic waves is approximately $25 \%$ higher than that in an ambient environment. Besner et al. [10] used a femtosecond laser for ablation in a vacuum, air and water for surface modifications; they showed that the threshold values of the Si and Au target materials were almost identical in all three environments. The values of $\mathrm{Si}$ in the single and multi-pulse irradiation regime were 0.4 and $0.2 \mathrm{~J} / \mathrm{cm}^{2}$; for $\mathrm{Au}$, their values were 0.9 and $0.3 \mathrm{~J} /$ $\mathrm{cm}^{2}$, respectively. Patel et al. [11] showed that the laser-ablation process is more efficient in water than in air, which depends on the thermal properties of the materials. It was also concluded that laser-ablation production in water is more suitable for the production of uniform nanoparticles and the mass production of nanoparticles. In addition to the aforementioned environments for laser ablation, Lindley et al. [12] studied the laser-ablation plume of an aluminium target material in a plasma environment, as well as in a vacuum and argon gas. It was concluded in this study that the laser-ablation plumes in the plasma expand and dissipate slightly faster than in the gas and the vacuum. Iqbal et al. [13] showed the effects of the laser fluence and ablation environments (vacuum and hydrogen) on the micro- and nanostructure of a Ge target material. It was shown in their study that the formation and growth of laser-induced periodic surface structures (LIPSS), cones and micro-bumps strongly depended on the laser fluence and environmental conditions. Hence, the growth, size and shape of these structures strongly depended on the laser fluence.

In this chapter, a critical comparison of laser ablation in different environments such as a vacuum, ambient air, different liquid environments and different background gases is presented. The optimal medium and laser-beam parameters for laser ablation will be designated.

\section{Laser-material interaction}

During laser-material interaction, the product materialises directly after laser irradiation onto the surface of the solid target material by condensing the plasma plume. After laser-pulse deposition onto the surface of the target material, the laser-pulse energy will heat the target, leading to an increase in the temperature of the materials. The temperature propagates in the axial and radial directions in a specific area. In the case of a perpendicular laser beam on a flat target material, the temperature propagation as a function of time $(t)$ and depth $(x)$ can be written as follows [14]:

$$
\Delta T(x, t)=2(1-R) \alpha I_{o}\left(\frac{t}{\pi k p c}\right) \operatorname{ierfc}\left(\frac{x}{2 \sqrt{k t / \rho c}}\right)
$$


where $t, R, \alpha, I_{\mathrm{o}}, k, \rho$ and $c$ are irradiation time, reflectivity, absorptivity, spatial distribution of laser intensity, thermal conductivity, target material density and velocity of light, respectively. When $x>2 \sqrt{k t / \rho c}$ the surface temperature can be simplified as follows:

$$
\Delta T(t)=\frac{2 \alpha I_{o} \sqrt{t}}{\sqrt{\pi k \rho c}}
$$

Two main things which have an effect on the laser ablation of materials are: laser-beam parameters such as laser fluence, laser wavelengths and laser-pulse duration, and factors in the experimental set-up, such as the type of environment and the solution. Laser-ablation mechanisms and their products are different depending on whether the laser-material interaction is produced by nanosecond, picosecond or femtosecond lasers [4]. In the laserablation process, groove width and depth are two important factors with which to characterise the results (see Figure 1) [15].

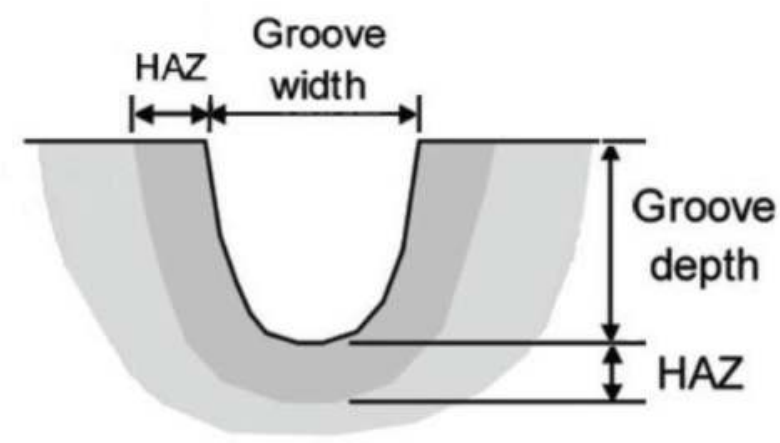

Figure 1. Groove width, groove depth and heat-affected zone of a laser-ablated material target.

Laser ablation in the target material occurs when the laser intensity reaches its threshold value. The laser-intensity threshold for material removal $\left(I_{\text {th }}\right)$ can be written as follows [15]:

$$
I_{\text {th }}=I_{o} \exp \left(\frac{-2 G_{w}^{2}}{d_{b}^{2}}\right)
$$

where $I_{o}, G_{w}$, and $d_{b}$ are laser-beam intensity, groove width and laser-beam diameter, respectively. This equation can be written as follows:

$$
\ln \left(\frac{I_{o}}{I_{\text {th }}}\right)=\left(\frac{2 G_{w}^{2}}{d_{b}^{2}}\right)^{\beta}
$$


where $\beta$ is an empirical coefficient which may be added to the formula due to some effects, such as recoil pressure, plasma-shielding effect, dynamics of vaporisation and multiple reflection of the laser beam in the cut channel. Thus, the groove width $\left(G_{w}\right)$ equation can be written as a general equation as follows:

$$
G_{w}=\sqrt{\frac{d_{b}^{2}}{2}\left[\ln \left(\frac{I_{o}}{I_{t h}}\right)\right]^{1 / \beta}}
$$

As shown in the following equation, the predictive model for groove depth $\left(G_{d}\right)$ can be calculated based on 'Furzikov's study and the $b$ coefficient applied to the logarithmic function of the laser intensity ratio' [15]:

$$
G_{d}=\left[\gamma \frac{\lambda}{\sqrt{n}}\left(\frac{1}{\alpha}+\sqrt{\frac{4 k d_{b}}{v}}\right)\right]^{1 / 2}\left[\ln \frac{I_{o}}{I_{t h}}\right]^{1 / \beta}
$$

where $\gamma$ is another empirical coefficient, $\lambda$ is the laser wavelength, $n$ is the refractive index of the target material, $\alpha$ is the absorption coefficient of the target material, $k$ is the thermal diffusivity of the target material and $v$ is the laser-traverse speed. The last empirical coefficient $(\gamma)$ is related to the degree of laser absorption and plasma formation in different environments such as air, water and ethanol, in which their penetration depths to produce a cut are different. In polymethyl methacrylate (PMMA) the approximated values of the refractive index $n$, absorption coefficient $\alpha$ and the thermal diffusivity $k$ are $1.4827,500 \mathrm{~cm}^{-1}$, and $1.073 \times 0^{-7} \mathrm{~m}^{2} / \mathrm{s}$. The HAZ formula can be written as [15]:

$$
G_{H A Z}=\sqrt{\frac{d_{b}^{2}}{8}\left[\ln \left(\frac{I_{o}}{I_{t h}}\right)\right]^{1 / \beta}}-\frac{G_{w}}{2}
$$

The groove formed by laser ablation of a Si target material in air and water is quite different. In water, the hole edge is very smooth, but in an ambient environment, the hole has bumps with a height of $3.6 \mathrm{~mm}$. In addition, the hole diameter in water is larger than that in air, and the hole depth in water is several times greater than in air [8]. Figure 2 shows the laser-ablated groove profile of a Silicon target material ablated in air and in water [8].

The ablation depth increases with increasing laser power, reducing spot size and decreasing scan speed [7]. In addition, the ablation rate significantly decreases with the depth of the hole [16].

During laser ablation, some of the laser energy will be lost in the ablation environment before it reaches the target material. The ratio of loss is higher in water than in air and in air, it is higher than in a vacuum. As shown in Figure 3, under the same laser-beam parameters the 
energy loss in air is higher than that in a vacuum. This is because for laser ablation in a vacuum, laser-induced air breakdown and ionisation do not exist; in addition, the ejected energetic electrons freely diffuse in the vacuum. The absorption of the laser beam which causes laser energy loss in the vacuum may only be caused by hot electron. Furthermore, absorption rate in a vacuum is very low because the collision frequency is low and the electron density is small [17]. In contrast, in air, laser-induced air breakdown does exist; as a result, laser absorption is increased. In water, laser absorption will be increased considerably due to the water level above the sample.
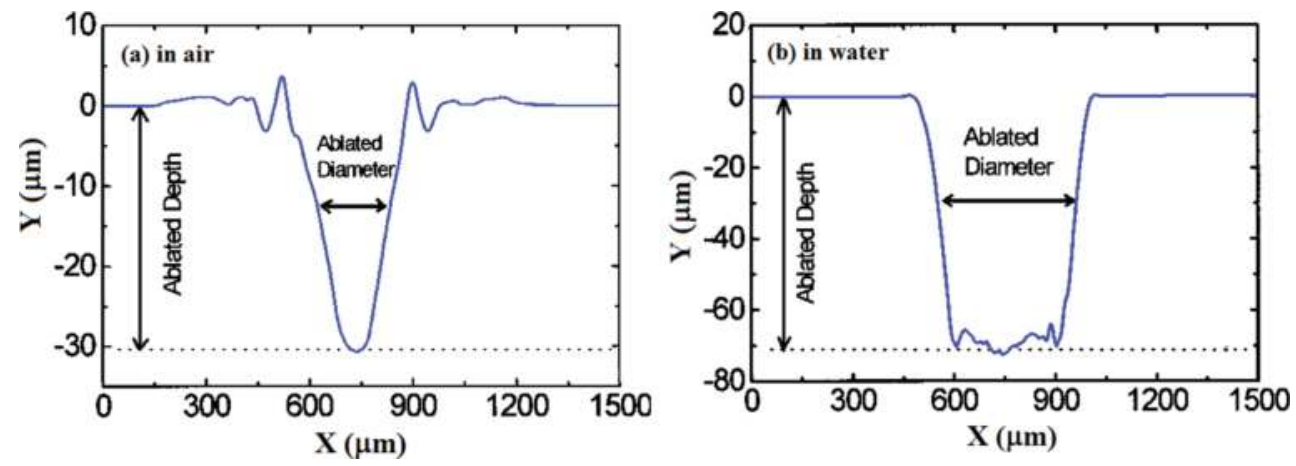

Figure 2. Laser-ablated region profile of a Si target after 1000-pulse irradiation in air (a) and water (b).

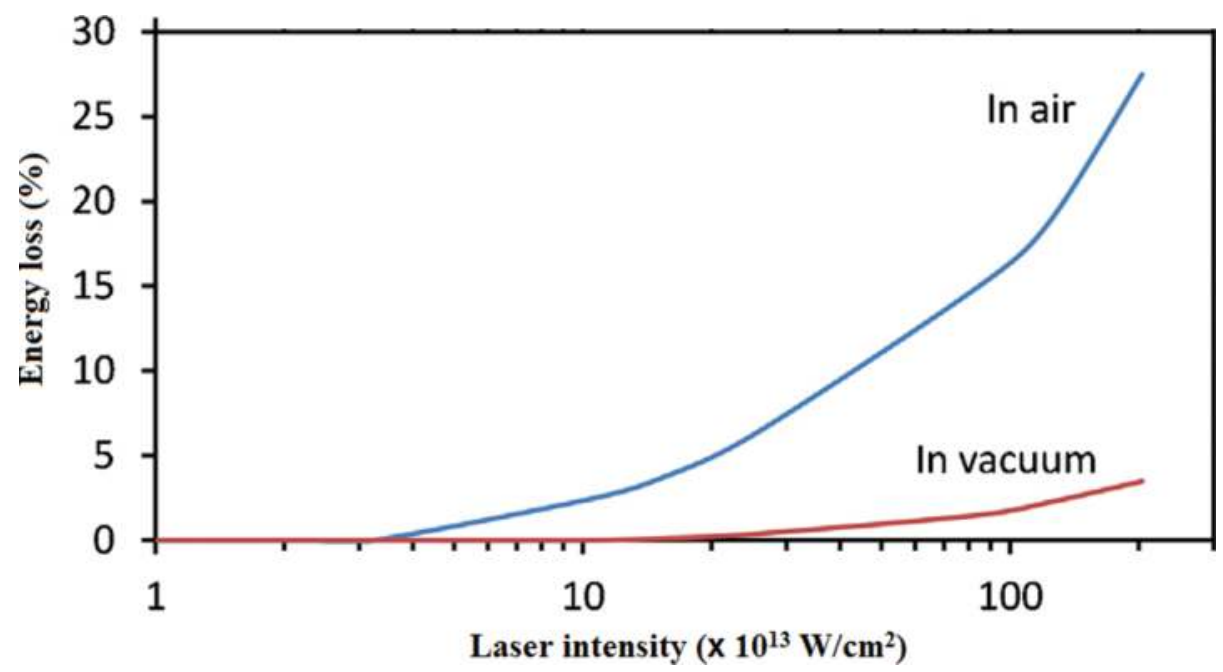

Figure 3. Pulsed laser-beam energy loss during laser ablation of aluminium target materials in air and in a vacuum. Laser wavelength $(\lambda)$ was $800 \mathrm{~nm}$ and laser pulse duration $(\tau)$ was $100 \mathrm{fs}$. 


\section{Laser ablation in a vacuum}

Laser ablation has been used in a vacuum using a vacuum chamber. A vacuum chamber is an empty rigid enclosure free from air and any gases, which are removed using a vacuum pump. As a result, a low-pressure environment is produced in the chamber. Laser ablation in a vacuum has been used to avoid any contamination during laser-material processing.

Laser-material interaction in a vacuum produces plasma at the surface of the target material, which expands considerably in the vacuum chamber [18]. An important factor in pulsed-laser ablation in different environments is the surface temperature of the target material. Figure 4 shows the temporal variations of the surface temperature $\left(T_{\mathrm{w}}\right)$ and equilibrium vapour density $\left(n_{\mathrm{s}}\right)$ above the surface of a bulk flat material of pure niobium $(\mathrm{Nb})$ in a vacuum chamber at a base pressure of $10^{-5} \mathrm{~Pa}$. The target material was irradiated using a Nd:YAG laser pulse at the $1064 \mathrm{~nm}$ wavelength. It can be noted that the maximum values of both $T_{\mathrm{w}}$ and $n_{\mathrm{s}}$ were reached at $0.3 \tau_{L}(4 \mathrm{~ns})$ at the maximum laser pulse [19]. It is worth mentioning that the temperature profile is not only a function of the laser-beam parameters but is also a function of the type of target material.

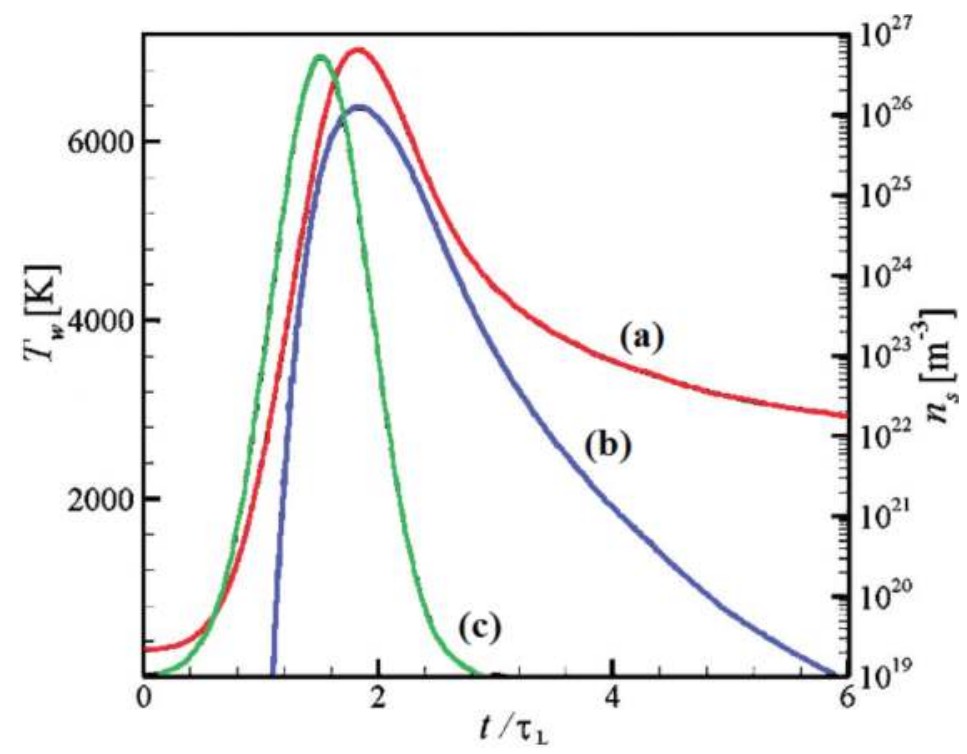

Figure 4. (a) The surface temperature, $T_{w^{\prime}}$ (b) equilibrium vapour density, $n_{\mathrm{s}^{\prime}}$ as a function of time and (c) irradiated pulsed laser.

The temperature profile of laser-material interaction in the vacuum can also be shown as a function of plume density. Figure 5 shows the temperature-density phase diagram of an aluminium (Al) foil target material irradiated by a femtosecond laser (wavelength: $800 \mathrm{~nm}$ and pulse duration: $100 \mathrm{fs}$ ) in a vacuum chamber at a backing pressure of $10^{-7}$ mbar [20]. The 
corresponding temperature $(T)$ of the plasma from which the ions originated, which were estimated for the IR and UV laser wavelengths, is about $10^{6}$ and $10^{5} \mathrm{~K}$, respectively [18].

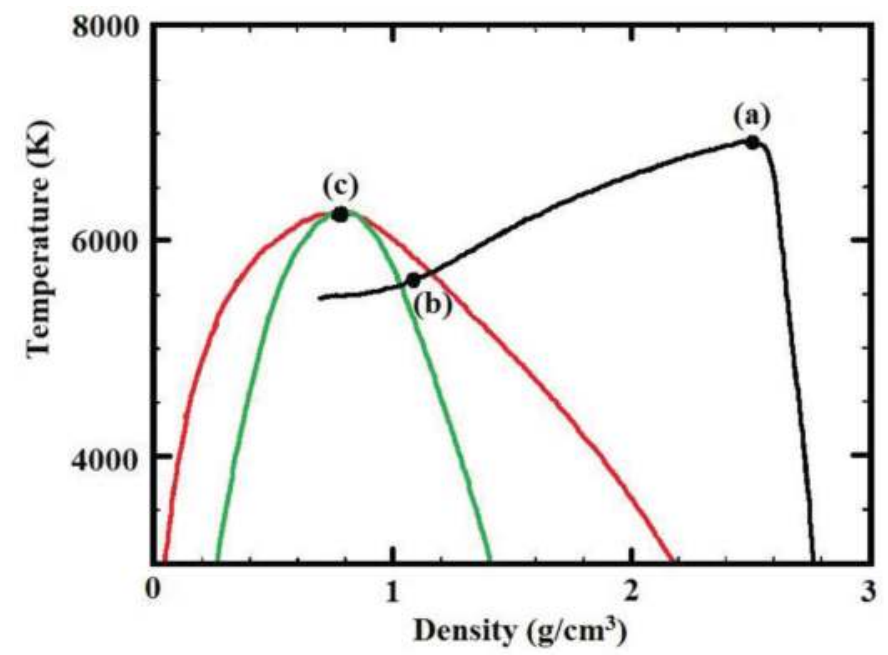

Figure 5. Diagram showing temperature as a function of plume density of Al foil target material irradiated by a femtosecond laser in a vacuum. The solid line represents the thermodynamic path of the Al absorbing volume (at a laser fluence of $400 \mathrm{~mJ} / \mathrm{cm}^{2}$ ). The dashed line (binodal) is the location of liquid-gas equilibrium states and the dotted line (spinodal) curve defines the limit of the homogenous phase. (a) and (b) are a particular case representing the physical conditions reached by the $\mathrm{Al}$ plume about 5 and 7 ps after the $100-\mathrm{fs}$ beings laser pumping, respectively. (c) indicates the critical point.

After laser-material interaction in the vacuum, the debris materials from the expanding plume have different speeds. The temporal profile of the emission intensity for nanoparticles and atoms, measured $5 \mathrm{~mm}$ from the surface of the target material at a laser fluence of $0.8 \mathrm{~J} / \mathrm{cm}^{2}$, shows that the light atoms fly faster than the nanoclusters or nanoparticles. It is shown that the average velocities of the nanoparticles and atoms are about $12 \times 10^{3}$ and $1 \times 10^{3} \mathrm{~m} / \mathrm{s}$, respectively [20]. Time-of-flight (TOF) measurements showed that the average velocity of the ion emission from a Cu material target in a vacuum by infrared (IR) (1064 nm wavelength) and ultraviolet $(\mathrm{UV})(308 \mathrm{~nm})$ are different and about $4.7 \times 10^{4}$ and $2.3 \times 10^{4} \mathrm{~m} / \mathrm{s}$, respectively [18].

The ablation depth (ablation depth per pulse) and emission yield as a function of the laser fluence at low and moderate laser fluence (about $150-500 \mathrm{~mJ} / \mathrm{cm}^{2}$ ) show logarithmic dependence. On the other hand, at higher laser fluence $\left(>500 \mathrm{~mJ} / \mathrm{cm}^{2}\right)$ the laser ablation sharply increased [20]. The laser ablation threshold in terms of the laser power density at 1064 and $308 \mathrm{~nm}$ laser wavelengths are about 7 and $3 \mathrm{~J} / \mathrm{cm}^{2}$, respectively [18].

In the vacuum, it was shown that the nanoparticles are directly generated from the target material by phase explosion. The condensation processes in the gas phase in the first stages of the plume expansion is not a means of producing nanoparticles in the vacuum [20]. It has been shown that during femtosecond laser interaction with tungsten to produce nanoparticles in a 
vacuum, the atomic plume emission acquired about $400 \mathrm{~ns}$, but the nanoparticle plume acquired about $100 \mu$ s after the ablating laser pulse [21].

\section{Laser ablation in air}

Another environment that has been used in laser-material processing is air. In general, air is the name given to the Earth's atmosphere. In the case of these experiments, it means that laser ablation will be carried out in an environment in which about $78 \%$ is Nitrogen $(\mathrm{N})$ and $21 \%$ is Oxygen (O). Laser ablation in air plays a substantial role in deep holes but has a low effect on initial surface ablation rates [16].

In general, ablation rate depends on laser fluence, repetition rate and the number of laser pulses [22]. It has been shown that the ablation rate in air at a laser fluence of less than $5.9 \mathrm{~J} \mathrm{~cm}^{-2}$ sharply increases and then slowly increases up to $40.7 \mathrm{~J} \mathrm{~cm}^{-2}$; after this point, the ablation rate decreases. The ablation rate also drops at a high repetition rate. The drop in ablation rate in both cases is thought to be due to attenuation of the laser energy caused by particle and plasma shielding, produced due to interactions with the remaining laser-generated particles on the ablated crater [22]. The ablation rate at reduced pressure in air shows that the averaged ablation rate increases with decreasing pressure from 1000 to about 250 mbar, regardless of the laser fluence and type of target material [23].

\section{Laser ablation in background gases}

Laser ablation in different background gases such as $\mathrm{He}, \mathrm{Ne}, \mathrm{Ar}, \mathrm{Kr}, \mathrm{Xe}$ and $\mathrm{N}_{2}$ has been used to improve the cutting-edge quality of laser ablation [24]. Heavier background gases produce a slower expansion, or more confinement of the vapour plume [3]. The gas environment has an effect on ablation efficiency. The improvement in laser-machining in different gases has been found to correlate to their potential ionisation [25].

As shown in Figure 6, the surface temperature increases due to the laser (photon) influence to a maximum of about $7000 \mathrm{~K}$ at $8 \mathrm{~ns}$. This corresponds to the maximum laser irradiance time profile. When the laser pulse is finished, the surface temperature reduces sharply to about $3000 \mathrm{~K}$ after $20 \mathrm{~ns}$, after which point, it gradually drops to about $1100 \mathrm{~K}$ at $100 \mathrm{~ns}$. It has been shown that the maximum surface temperatures for $\mathrm{He}, \mathrm{Ne}, \mathrm{Ar}, \mathrm{Kr}$ and $\mathrm{N}_{2}$ are 7088, 7062, 7036, 7025 and $7037 \mathrm{~K}$, respectively. It can be concluded that the surface temperature increases slightly with decreasing mass and increasing ionisation potential of the background gas [3].

Concerning the generation of nanoparticles in a background gas, Nichols et al. [26] produced Ag nanoparticles by laser ablation in argon, nitrogen and helium at a variety of gas pressures. It was concluded that by selecting an appropriate gas type and pressure, Ag nanoparticles can be produced and controlled in the range of 4-20 nm. In addition, the smallest Ag nanoparticles (with a mean diameter of $5 \mathrm{~nm}$ ) were produced in helium gas at $1 \mathrm{~atm}$ and below, and the 


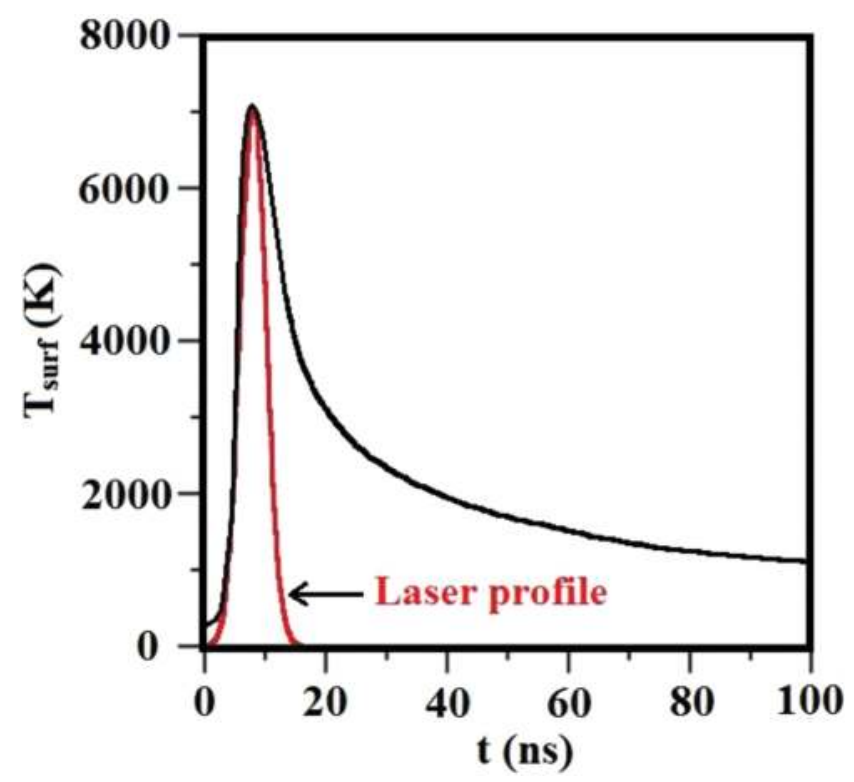

Figure 6. Calculated surface temperature $\left(T_{\text {surf }}\right)$ and laser irradiance-time profile as a function of time $(t)$ for a laser of $266 \mathrm{~nm}$, with $5 \mathrm{~ns}$, and an irradiance of $1 \mathrm{GW} / \mathrm{cm}^{2}$.

largest nanoparticles (with a mean diameter of $19 \mathrm{~nm}$ ) were produced in nitrogen gas. Furthermore, the average size of the nanoparticles increases with increasing molecular weight of the gas. The kinetic energy of the ablated atoms in a gas background and the width of their angular distribution has also been found to decrease with increasing ambient pressure [27].

\section{Laser ablation in liquid environments}

The use of pulsed-laser ablation at the solid-liquid interface was first reported by Patil and coworkers in 1987 to produce a metastable form of iron oxide from a pure iron target material [28]. Laser ablation in liquids has been used to produce nanoparticles as an alternative to chemicals because ablation in liquid is considered a cleaner environment in which to produce nanoparticles. Different liquids have different effects on the production of nanoparticles. For example, laser ablation of a Tin (Sn) target in water produces polycrystalline tin dioxide $\left(\mathrm{SnO}_{2}\right)$ nanoparticles, while ablation in ethanol produces single crystals of tin coated with tin hydroxide $\left(\mathrm{Sn}(\mathrm{OH})_{2}\right)$ nanoparticles [29].

The generation of nanoparticles in different solutions, particularly in pure water or deionised water, has received much attention from researchers in the field of nanoparticle generation because pure water is a suitable environment for the synthesis of nanoparticles and is free from any contamination. 
Many number of research studies have been published on the production of several types of nanoparticle using laser ablation in deionised water [30-36], sodium dodecyl sulfate (SDS) [35, 37-40], acetone [41, 42], ethanol and ethylene chloride [33, 43, 44], polyvinylpyrrolidone (PVP) $[45,46]$ and liquid nitrogen solutions (LN) [47]. Both pulsed-laser beams [31, 33, 36-39, 47] and continuous-wave (CW) laser beams [30,48-56] were used to produce nanoparticles.

It has been shown that the ablation rate of Si varies with the water level above the target material. It has been concluded that the laser-ablation rate can be considerably enhanced by using a water level of $1.1 \mathrm{~mm}$ [57].

Laser ablation of solids in liquids is an effective technique with considerable potential in the generation of nanocrystals, which allows multilateral design through choosing appropriate solid target materials and confining liquids [58]. The response of different liquid solutions in the generation of nanoparticles varies considerably. Considering the generation of $\mathrm{ZnO}$ nanoparticles by laser ablation, in a cetrimonium bromide (CTAB) solution, the highest ablation rate and highest crystallinity of nanoparticles were observed. The largest nanoparticles were produced in acetone. In addition, in a CTAB solution, the morphology of $\mathrm{ZnO}$ nanoparticles changed. However, the number of $\mathrm{Zn} @ \mathrm{ZnO}$ core-shell nanoparticles was found to be larger in CTAB and SDS. In general, cationic surfactants have been found to considerably change the shape of $\mathrm{ZnO}$ nanoparticles. The morphology of $\mathrm{ZnO}$ nanoparticles in SDS, acetone and water was found to be spherical, while in CTAB was spindle-like [59].

\section{Comparison of laser ablation and nanoparticle production in different environments}

Conducting laser ablation in different environments leads to different ablation mechanisms, ablation rate, ablation profile and efficiency. In addition, laser-generation of nanoparticles in various media produces different nanoparticles in terms of their morphology, size and size distribution. In the following sections, the compassion of laser ablation and nanoparticle production in different environments in terms of ablation mechanism, ablation rate, ablation efficiency and their differences and advantages will be presented.

\subsection{Ablation mechanisms in different environments}

Laser ablation is an important process in laser-material processing to obtain precision machining or to produce controllable nanoparticles in terms of size, size distribution and morphology. The laser-ablation mechanism various in different environments; for example, in the case of laser ablation in a liquid environment, when a target material is irradiated in a liquid environment by a pulsed laser, the laser energy will be absorbed by the target, which heats it, causing the target to reach melting temperature and evaporation. As a result of this, the material will be ablated from the surface of the target into the liquid solution, and finally nanoparticles are produced as a result of condensation and nucleation. The water level above the sample has different effects on the laser ablation: the main effect is decreasing the laser 
power for some laser wavelengths, such as at $1064 \mathrm{~nm}$. Furthermore, the focal length will change as it passes through the liquid medium.

In femtosecond-laser ablation of an aluminium target material, it has been shown that the ablation (material ejection) mechanism and shock-wave evolution are different in water and in air. In the case of a liquid environment, the ejected materials are confined to a small area near the surface of the target material. Furthermore, the liquid media significantly decreases the velocity of the shock waves close to the velocity of sound within nanoseconds, decelerating slowly afterwards. However, in the case of air, the ejected material can propagate away from the target material with a low resistance within the shock waves [60]. Different liquid environments such as deionised water and methanol produce different sizes of nanoparticles in laser generation of nanoparticles [61]. Small Au nanoparticles were produced by pulsed-laser ablation in sodium dodecylbenzene sulfonate (SDBS) in comparison with those produced in sodium dodecyl sulfate (SDS) surfactant due to SDS having smaller molecules than SDBS [62].

There is a considerable difference in the ablation mechanism and thermal damage of laser ablation in air and in water. No thermal damage has been observed in a water environment, whereas considerable thermal damage has been documented in air. Thermal damage has been found to increase with an increase in the number of laser pulses. In addition, in the case of machining in air, the ablated surface is smooth, while in the case of underwater machining, the surface is rough. In the later case, the surface shows molten materials rapidly solidified with a dendritic structure. Consequently, the ablation depth is more affected by laser fluence in the case of underwater machining than in ambient air. In contrast, at low laser fluence, the ablation depth is significantly higher in ambient air than in water [63]. All these differences are due to the use of different ablation mechanisms in different environments.

In a vacuum, the time taken for energy to be transferred from the bulk target material to the surface, which produces the high-energy tail, exceeds other characteristic timescales such as surface cooling time and the electron-ion temperature equilibration time. In air, the interaction between the gas atoms and the surface significantly reduces the lifetime of this non-equilibrium surface state, which allows thermal evaporation to take place before the surface cools. The ablation threshold in air is lower by half than that in a vacuum. This is because in air, after the laser pulse, thermal evaporation is responsible for the lower ablation threshold, while in a vacuum, the threshold corresponds to non-equilibrium ablation during the laser pulse [64]. The plasma produced in air was found to be much hotter and denser than that produced in a vacuum [65]. It was shown that the breakthrough times of laser ablation for drilling in a vacuum are slightly lower for stainless steel and much lower for aluminium than in air. In addition, shorter laser pulses in the range of $150 \mathrm{fs}$ to 20 ps drill faster than 500 ps pulses [16].

It has also been shown that the dynamic process of femtosecond laser ablation of silicon (Si) in a vacuum is considerably different from that in air. In the case of the laser ablation in air at under $1 \times 10^{5} \mathrm{~Pa}$, all the expected processes, such as the shock-wave front, the nearly concentric semicircular stripes and the contact front were observed, while in the case of laser ablation in a vacuum, these phenomena were not observed. In addition, another significant factor in the dynamic process of femtosecond laser ablation is the ambient air around the target, which acts as an effective heating dissipation factor and a stagnation force [66]. 


\subsection{Ablation rate in different environments}

The rate at which materials are ablated is also different in different environments. This may be due to different ablation mechanisms and ablation thresholds. Ben-Yakar and Byer [67] showed that in the case of single-shot laser ablation, the ablation threshold in air is lower than that in a vacuum. The reason for this may be due to the modified absorption process because air may accelerate the ablation chemistry; due to air resistance, the hot plasma expands at a slower speed in comparison with that in a vacuum. On the other hand, in the case of multiple laser pulses overlapping, the resulting incubation effect reduces dependence on the processing environment.

In air, ablation rate decreases with ablating deep holes because of the non-linear effect. The non-linear effect of laser ablation in air is due to localising the laser beam. This means that ablation products cannot escape the holes, which causes self-focusing of the laser pulses even at a power less than the critical power for self-focusing in ambient air. Based on these effects, it has been shown that the ablation efficiency in air is slightly higher than that in a vacuum (0.51 and $0.43 \%$, respectively) [67].

The effects of using a buffer gas on the ablation rate of $\mathrm{Ti}, \mathrm{W}, \mathrm{Fe}, \mathrm{Cu}$ and Mo target materials at different fluencies have been studied. In general, the effect of He gas at 1000 mbar and air at 1 mbar is quite similar, so in both cases, the averaged ablation rate slightly increased; this effect is more acute with increasing laser fluence. In the case of Ar gas, the average ablation rate decreased because the absorption of the laser beam by the plasma is more considerable in comparison with this in He or in ambient air. This effect was found to be independent of the laser fluence. As a result, 'more absorption of the laser energy reaching the sample necessarily implies that less sample is vaporized' [23].
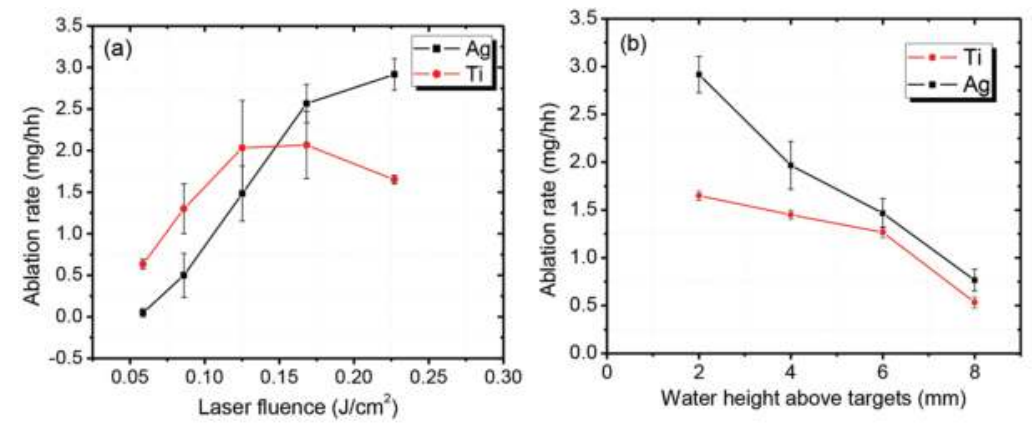

Figure 7. (a) The ablation rate of Ag and Ti target materials as a function of laser fluence at a fixed water level (2 mm), and $(b)$ as a function of water level at a fixed laser fluence $\left(0.22 \mathrm{~J} / \mathrm{cm}^{2}\right)$.

Figure 7a shows the ablation rate of $\mathrm{Ag}$ and $\mathrm{Ti}$ target materials as a function of laser fluence in deionised water at a fixed water level above the targets. A picosecond laser at $\lambda=1064 \mathrm{~nm}$, $f=200 \mathrm{kHz}, v=30 \mathrm{~mm} / \mathrm{s}, t=1 / 2 \mathrm{~h}$ and a laser spot size $=125 \mu \mathrm{m}$ was used to ablate the materials in deionised water. At lower laser fluencies, the ablation rates of both samples increased 
slightly, but at higher laser fluencies the ablation rates decreased. It can be seen that the ablation rate of the Ti target decreases significantly when the laser fluence increases above $0.15 \mathrm{~J} / \mathrm{cm}^{2}$. This is because of the reduction in laser pulse energy reaching the target material, which occurs after increasing laser-beam absorption and scattering due to higher plasma/plume density [4]. The ablation rate of the nanoparticles in liquid environments is also a function of the scan speed of the laser beam. This decreased with an increase in scan speed because at the beginning, higher scan speeds produce a high amount of nanoparticles, leading more nanoparticles to disperse in the solution, which rapidly prevent the laser pulse energy from reaching the target material. In other words, at high scan speeds, there is not enough time for nanoparticles to disperse in the solution, but at low scan speeds, there is enough time for nanoparticles to disperse, allowing the laser energy to reach the target almost completely. It has been shown that nanoparticles produced in deionised water would travel towards the laser beam which causes the laser beam to scatter and prevent it from reaching the target material [68].

In the case of laser ablation in deionised water, the ablation rate of materials (Ag and Ti targets) decreased as the water level above the samples increased at fixed laser fluence. Broadly speaking, the ablation rate decreased with increasing water level and the rate of decrease of $\mathrm{Ag}$ was found to be faster than that of Ti. It was also shown that the amount of Ag materials or nanoparticles ejected was greater than the amount of Ti nanoparticles (see Figure 7b) [4].

Recently, Hamad et al. [69] produced nanoparticles in ice water by picosecond laser ablation; it was shown that the ablation rate in ice water is lower than that produced in deionised water because of the higher attenuation coefficient and absorptivity of the ice water (frozen deionised water) to the laser-beam wavelength in comparison with unfrozen deionised water. The ratio of the ablation rate in ice water and deionised water was about 1:3.

\subsection{Ablation efficiency in different environments}

It was expected that the ablation efficiency would be lower in a vacuum than that in air because in a vacuum less work is required for the hydrodynamic expansion of plasma in a non-resistant environment. However, a larger groove diameter can be obtained in air because the single-shot ablation threshold is lower in air. In addition, the ablation volume is slightly higher in air than in a vacuum because the ablation depth is determined by the optical penetration depth, which is independent of the processing environment [67]. The non-linear dependence of the ablation rate or productivity on the laser fluence in a vacuum with femtosecond-laser ablation of nickel was also observed [70].

It has been reported that the laser-ablation efficiency of nanosecond-laser pulses is similar in both a liquid environment and ambient air, but in the case of laser ablation by femtosecondlaser pulses, the ablation efficiency was higher in air in comparison with that in water [32]. The mass rate of nanoparticle generation in air (aerosol) was observed to be 100 times higher than in water (colloid) [71]. The laser-ablation efficiency was also influenced by the laser-spot size and the irradiation time [72]. It was also reported that the laser-ablation efficiency in the vacuum decreased with increasing laser fluence, caused by increasing the duration of the atomisation stage $[73,74]$. Although laser ablation of materials depends upon their thermal properties, laser ablation is more effective in a liquid environment that in air. In addition, in 
air, the microparticles were notably distinct both near and far from the crater on the brass target. However, in water, the narrow distribution of nanoparticles away from the crater was also sound. It was concluded that water is a more suitable environment in which to produce uniform and large quantities of nanoparticles by laser ablation [11]. Hermann et al. [74] determined that the ablation depth of copper and gold in a vacuum chamber increased gradually at low laser fluence up to $0.5 \mathrm{~J} / \mathrm{cm}^{2}$, but that this figure increased considerably at high laser fluence.

The laser-ablation process is also used to clean the surfaces of materials. The effectiveness of laser-ablation cleaning methods on silver artefacts in different media (air, water and a vacuum) was characterised. Before cleaning the samples, they were treated with $\mathrm{HCl}(37 \%)$ solutions for several hours to simulate the formation of a chloride patina $(\mathrm{AgCl})$ on marine archeological silver artefacts. It was shown that the patina was removed from a few to $300 \mu \mathrm{m}$ in air and in water and removed completely under vacuum conditions. However, the formation of a white patina in air and water and an increasing amount of oxygen on the surface of the samples were observed during cleaning process. This situation shows that 'in ambient reach in oxygen laser ablation favourite the oxygen absorbance on surface and the formation of a thin layer of AgO which dulls the surface' [75]. This hypothesis is proven by unobserving surface tarnishing in a vacuum during laser ablation. It can be concluded that the optimal conditions in which to clean silver artefacts is under vacuum conditions [75].

\subsection{Differences and advantages of different environments to laser ablation and generation of nanoparticles}

Many important differences have been observed between laser ablation in a vacuum, air, gas and liquid environments. These differences can be summarised as follows: first, in water, at the liquid-solid interface, a plasma plume is created. In a liquid solution, the ablated materials and plasma plume expansion are confined, which leads to the production of a high temperature and pressure caused by the mechanical effects. Second, chemical reactions are produced at the interfaces between the laser-induced plasma and the liquid and in the laser-induced plasma at high temperature and pressure. Third, in water, the quenching time of the plasma plume is much shorter than in gas and a vacuum [57].

The advantages of using laser ablation in liquid environments to produce nanomaterials over other methods such as chemicals are as follows: (i) simple and clean production because the process does not need a catalyst and no byproducts are formed; (ii) the process does not require extreme temperature and pressure; (iii) the method leads to the production of a new phase of nanocrystals which may occur in both liquids and solids, which presents more options to choose and combine interesting and desired solid targets and liquids to produce nanocrystals and nanostructures of new compounds or bimodal [57]. Another advantage of using liquid environments in the production of nanoparticles is the ability to use the liquid as a medium in which to collect the nanoparticles. In addition, it has also been shown that the application advantages of nanoparticles produced in liquid environments in comparison with conventionally generated nanoparticles are the following: (i) the number of bimolecules which can be conjugated on the surface of laser-produced nanoparticles in water is three to five times higher 
than their chemically produced counterparts; (ii) pulsed-laser ablation in a polymer solution allows doping or embedding of nanoparticles into matrices of the polymer for quick nanomaterial prototyping; (iii) production of nanoparticles in liquid environments via pulsed-laser ablation generates pure and aggregated nanoparticles. Using aggregated nanoparticles as a substrate in surface-enhanced Raman scattering (SERS) supplies higher signal-to-noise ratio [76].

The advantages of performing laser ablation in liquid environments over vacuum chambers are simple experimental set-up; low price of liquids and higher availability; easy transportation and storage; no chemical precursors; no chemical contamination resulting in $100 \%$ pure nanoparticles. In other words, more nanoparticle sites or surface atoms are available for drug loading, fictionalisations and environmental sensing. Furthermore, laser ablation in liquids produces charged nanoparticles which form high-stability colloidal nanoparticles. In addition, nanoparticles produced in liquid solution are not inhalable, unlike the dry nanopowders generated in gas-phase pulsed-laser ablation [76].

Yang [57] showed that laser ablation of solids in confined liquid environments is more effective than in a vacuum and diluted gas. This is because in liquids, the solid target material continuously etches at the plasma-solid interface due to the high temperature, high pressure and high density of plasma, as a result, promoting the total ablation rate.

\section{Conclusions}

Laser ablation in laser-material processing has been carried out in different environments such as a vacuum, ambient air, different liquid environments and different background gases. Each medium presents different responses to laser ablation; in other words, different ablation mechanisms are observed in each environment. Although, generation of nanoparticles via pulsed-laser ablation of a solid target material in a vacuum chamber, air and gas have been widely developed, but the advancement of techniques performed in liquid environments has been of the greatest interest to researchers. The optimal medium for generation of nanoparticles is deionised water.

\section{Author details}

Abubaker Hassan Hamad ${ }^{1 *}$, Khawla Salah Khashan² and Aseel Abdulkreem Hadi²

*Address all correspondence to: abubaker.hamad75@yahoo.co.uk

1 General Science Department, Faculty of Education, Soran University, Soran City, Erbil, Kurdistan Region, Iraq

2 Division of Laser physics, Department of Applied Science, University of Technology, Baghdad, Iraq 


\section{References}

[1] Siraj, K., Y. Sohail and A. Tabassum, Turkish Journal of Physics, 2011. 35(2): p. 179-183.

[2] Nakamura, D., T. Tanaka, T. Ikebuchi, T. Ueyama, F. Nagasaki, Y. Fujiwara, M. Higashihata, H. Ikenoue and T. Okada. Ultraviolet lasing from spherical $\mathrm{ZnO}$ microcrystal produced by laser ablation in air. in 2015 20th Microoptics Conference (MOC). 2015: IEEE.

[3] Bogaerts, A., Z. Chen and Bleiner, D. Journal of Analytical Atomic Spectrometry, 2006. 21(4): p. 384-395.

[4] Hamad, A., L. Li and Liu, Z. Applied Physics A, 2015. 120(4): p. 1247-1260.

[5] Howard, K., S. Lawson and Y. Zhou, Welding Journal New York, 2006. 85(5): p. 101.

[6] Santo, L., International Journal of Surface Science and Engineering, 2008. 2(5): p. 327336.

[7] Ullmann, F., U. Loeschner, L. Hartwig, D. Szczepanski, J. Schille, S. Gronau, T. Knebel, J. Drechsel, R. Ebert and H. Exner. Highspeed laser ablation cutting of metal. in SPIE LASE. 2013: International Society for Optics and Photonics.

[8] Zhou, X., K. Imasaki, H. Furukawa, H. Umino, K. Sakagishi, S. Nakai and Yamanaka, C. Optics \& Laser Technology, 2001. 33(3): p. 189-194.

[9] Zhu, S., Y. Lu, M. Hong and Chen, X. Journal of Applied Physics, 2001. 89(4): p. 24002403.

[10] Besner, S., J.-Y. Degorce, A.V. Kabashin and M. Meunier. Surface modifications during femtosecond laser ablation in vacuum, air, and water. in Photonics North. 2004: International Society for Optics and Photonics.

[11] Patel, D., R.P. Singh and R.K. Thareja, Applied Surface Science, 2014. 288: p. 550-557.

[12] Lindley, R., R. Gilgenbach, C. Ching, J. Lash and Doll, G. Journal of Applied Physics, 1994. 76(9): p. 5457-5472.

[13] Iqbal, M.H., S. Bashir, M.S. Rafique, A. Dawood, M. Akram, K. Mahmood, A. Hayat, R. Ahmad, T. Hussain and A. Mahmood, Applied Surface Science, 2015. 344: p. 146-158.

[14] Jinghua Han and Yaguo Li (2011). Interaction Between Pulsed Laser and Materials, Lasers - Applications in Science and Industry, Dr Krzysztof Jakubczak (Ed.), ISBN: 978-953-307-755-0, InTech, Available from: http://www.intechopen.com/books/lasersapplications-in-science-and-industry/interaction-between-pulsedlaser- and-materials.

[15] Tangwarodomnukun, V. and Chen, H.-Y. Materials and Manufacturing Processes, 2015. 30(5): p. 685-691.

[16] Wynne, A. and Stuart, B. Applied Physics A, 2003. 76(3): p. 373-378. 
[17] Zhao, X. and Y.C. Shin, Applied Surface Science, 2013. 283: p. 94-99.

[18] Torrisi, L., S. Gammino, L. Andò, V. Nassisi, D. Doria and Pedone, A. Applied Surface Science, 2003. 210(3): p. 262-273.

[19] Bykov, N.Y., N. Bulgakova, A. Bulgakov and G. Loukianov, Applied Physics A, 2004. 79(4-6): p. 1097-1100.

[20] Amoruso, S., R. Bruzzese, M. Vitiello, N. Nedialkov and P. Atanasov, Journal of Applied Physics, 2005. 98(4): p. 044907.

[21] Harilal, S., N. Farid, A. Hassanein and V. Kozhevin, Journal of Applied Physics, 2013. 114(20): p. 203302.

[22] Shaheen, M., J. Gagnon and B. Fryer, Laser Physics Letters, 2015. 12(6): p. 066103.

[23] Vadillo, J., J. Fernandez Romero, C. Rodriguez and J. Laserna, Surface and Interface Analysis, 1999. 27(11): p. 1009-1015.

[24] Guo, H. and Q. Lou, Thin Solid Films, 1992. 218(1): p. 274-276.

[25] Chan, W., A.P. Leung, X. Mao and R. Russo, Applied Surface Science, 1998. 127: p. 269273.

[26] Nichols, W.T., G. Malyavanatham, D.E. Henneke, J.R. Brock, M.F. Becker, J.W. Keto and H.D. Glicksman. Journal of Nanoparticle Research, 2000. 2(2): p. 141-145.

[27] Gusarov, A., A. Gnedovets and I. Smurov, Applied Surface Science, 2000. 154: p. 66-72.

[28] Patil, P., D. Phase, S. Kulkarni, S. Ghaisas, S. Kulkarni, S. Kanetkar, S. Ogale and V. Bhide, Physical Review Letters, 1987. 58(3): p. 238.

[29] Musaev, O., M. Driver, E. Sutter, A. Caruso, J. Wrobel and M. Kruger, Applied Physics A, 2013. 113(2): p. 355-359.

[30] Tsuji, T., Y. Okazaki, T. Higuchi and M. Tsuji, Journal of Photochemistry and Photobiology A: Chemistry, 2006. 183(3): p. 297-303.

[31] Simakin, A.V., V.V. Voronov, G.A. Shafeev, R. Brayner and F. Bozon-Verduraz, Chemical Physics Letters, 2001. 348(3-4): p. 182-186.

[32] Tsuji, T., T. Kakita and M. Tsuji, Applied Surface Science, 2003. 206(1-4): p. 314-320.

[33] Dolgaev, S.I., A.V. Simakin, V.V. Voronov, G.A. Shafeev and F. Bozon-Verduraz, Applied Surface Science, 2002. 186(1-4): p. 546-551.

[34] Kazakevich, P.V., A.V. Simakin, V.V. Voronov and G.A. Shafeev, Applied Surface Science, 2006. 252(13): p. 4373-4380.

[35] Liang, C.H., Y. Shimizu, T. Sasaki and N. Koshizaki, Applied Physics A, 2005. 80(4): p. 819-822. 
[36] Tsuji, T., K. Iryo, Y. Nishimura and M. Tsuji, Journal of Photochemistry and Photobiology A: Chemistry, 2001. 145(3): p. 201-207.

[37] Mafuné, F., J.-y. Kohno, Y. Takeda, T. Kondow and H. Sawabe, The Journal of Physical Chemistry B, 2000. 104(35): p. 8333-8337.

[38] Mafuné, F., J.-y. Kohno, Y. Takeda, T. Kondow and H. Sawabe, The Journal of Physical Chemistry B, 2000. 104(39): p. 9111-9117.

[39] Chen, Y.-H. and C.-S. Yeh, Colloids and Surfaces A: Physicochemical and Engineering Aspects, 2002. 197(1-3): p. 133-139.

[40] Sasaki, T., C. Liang, W.T. Nichols, Y. Shimizu and N. Koshizaki, Applied Physics A, 2004. 79(4-6): p. 1489-1492.

[41] Tarasenko, N.V., A.V. Butsen and E.A. Nevar, Applied Surface Science, 2005. 247(1-4): p. $418-422$.

[42] Menéndez-Manjón, A., J. Jakobi, K. Schwabe, J.K. Krauss and S. Barcikowski, JLMNJournal of Laser Micro/Nanoengineering, 2009. 4(2): p. 95-99.

[43] Ganeev, R.A., M. Baba, A.I. Ryasnyansky, M. Suzuki and H. Kuroda, Optics Communications, 2004. 240(4-6): p. 437-448.

[44] Ohshima, T., S. Nakashima, T. Ueda, H. Kawasaki, Y. Suda and K. Ebihara, Thin Solid Films, 2006. 506-507(0): p. 106-110.

[45] Tsuji, T., D.H. Thang, Y. Okazaki, M. Nakanishi, Y. Tsuboi and M. Tsuji, Applied Surface Science, 2008. 254(16): p. 5224-5230.

[46] Tsuji, T., T. Mizuki, S. Ozono and M. Tsuji, Journal of Photochemistry and Photobiology A: Chemistry, 2009. 206(2-3): p. 134-139.

[47] Sasaki, K. and N. Takada, Pure and Applied Chemistry, 2010. 82(6): p. 1317.

[48] Liu, Z., Y. Yuan, S. Khan, A. Abdolvand, D. Whitehead, M. Schmidt and L. Li, Journal of Micromechanics and Microengineering, 2009. 19(5): p. 054008.

[49] Phuoc, T.X., Y. Soong and M.K. Chyu, Optics and Lasers in Engineering, 2007. 45(12): p. 1099-1106.

[50] Ramesh, P., S. Muthukkumarasamy, K. Dhanabalan, T. Sadhasivam and K. Gurunathan, Digest Journal of Nanomaterials and Biostructures, 2012. 7(4): p. 8.

[51] Zhang, Q., C. Sun, Y. Zhao, S. Zhou, X. Hu and P. Chen, Environmental Science \& Technology, 2010. 44(21): p. 8270-8275.

[52] Cozzoli, P.D., R. Comparelli, E. Fanizza, M.L. Curri, A. Agostiano and D. Laub, Journal of the American Chemical Society, 2004. 126(12): p. 3868-3879.

[53] Yu, B., K.M. Leung, Q. Guo, W.M. Lau and J. Yang, Nanotechnology, 2011. 22(11): p. 115603. 
[54] Nikov, R.G., A.S. Nikolov, N.N. Nedyalkov, I.G. Dimitrov, P.A. Atanasov and M.T. Alexandrov, Applied Surface Science, 2012. 258(23): p. 9318-9322.

[55] Liu, C., M. Hong, Y. Zhou, G. Chen, M. Saw and A. Hor, Physica Scripta, 2007. 2007(T129): p. 326.

[56] Khan, S., Y. Yuan, A. Abdolvand, M. Schmidt, P. Crouse, L. Li, Z. Liu, M. Sharp and K.G. Watkins, Journal of Nanoparticle Research, 2009. 11(6): p. 1421-1427.

[57] Yang, G., Progress in Materials Science, 2007. 52(4): p. 648-698.

[58] Wafaa Mustafa Aly Soliman, Fundamental Studies on the Synthesis Dynamics of Nanoparticles by Laser Ablation in Pressurized Water, a dissertation submitted in partial fulfillment of the requirements for the degree of doctor of engineering, department of electrical engineering and computer science, Nagoya Univeristy, Nagoya, Japan, March 2011.

[59] Moradi, M., E. Solati, S. Darvishi and D. Dorranian, Journal of Cluster Science, 2016. 27(1): p. 127-138.

[60] Hu, H., T. Liu and H. Zhai, Optics Express, 2015. 23(2): p. 628-635.

[61] Farahani, S.V., A. Mahmoodi and M. Goranneviss, International Nano Letters, 2016. 6: p. 5.

[62] Essaidi, A., B. Schöps, A. Aumman, S. Xiao, C. Esen and A. Ostendorf, Journal of Laser Micro Nanoengineering, 2013. 8(2): p. 131.

[63] Choo, K., Y. Ogawa, G. Kanbargi, V. Otra, L. Raff and R. Komanduri, Materials Science and Engineering: A, 2004. 372(1): p. 145-162.

[64] Gamaly, E.G., N. Madsen, M. Duering, A.V. Rode, V.Z. Kolev and B. Luther-Davies, Physical Review B, 2005. 71(17): pp. 174405.

[65] Liu, Y.H., X.D. Liu, M. Chen and M.W. Zhao. Laser ablation of Ti-Al alloy in vacuum and air environments. in Applied Mechanics and Materials. 2012: Trans Tech Publ.

[66] Wu, Z., N. Zhang, M. Wang and X. Zhu, Chinese Optics Letters, 2011. 9(9): p. 093201.

[67] Ben-Yakar, A. and R.L. Byer, Journal of Applied Physics, 2004. 96(9): p. 5316-5323.

[68] Hamad, A., L. Li, Z. Liu, X.L. Zhong, G. Burke and T. Wang, Applied Physics A, 2016. 122(4): p. $1-12$.

[69] Hamad, A., L. Li, Z. Liu, X.L. Zhong and T. Wang, Applied Physics A, 2015. 119(4): p. 1387-1396.

[70] Amoruso, S., R. Bruzzese, X. Wang, N. Nedialkov and P. Atanasov, Journal of Physics D: Applied Physics, 2007. 40(2): p. 331.

[71] Hahn, A., S. Barcikowski and B.N. Chichkov, Pulse, 2008. 40(45): p. 50. 
[72] Pyatenko, A., K. Shimokawa, M. Yamaguchi, O. Nishimura and M. Suzuki, Applied Physics A, 2004. 79(4-6): p. 803-806.

[73] Noël, S., J. Hermann and T. Itina, Applied Surface Science, 2007. 253(15): p. 6310-6315.

[74] Hermann, J., S. Noël, T.E. Itina, E. Axente and M.E. Povarnitsyn, Laser Physics, 2008. 18(4): p. 374-379.

[75] Stranges, F., M. Barberio, P. Barone, V. Pingitore, F. Xu and A. Bonanno, Journal of Earth Science and Engineering, 2012. 2(12): p. 697.

[76] Subhash Chandra Singh, Haibo Zeng, Chunlei Guo and Weiping Cai (2012).Lasers: Fundamentals, Types, and Operations. In, Nanomaterials: Processing and Characterization with Lasers (pp. 1-34), DOI: 10.1002/9783527646821.ch1. Copyright (C) 2012 Wiley-VCH Verlag \& Co. KGaA. 